\title{
Analysis of Sawdust by X-ray Photoelectron Spectroscopy
}

Guilin Jiang

Brigham Young University - Provo

Ghaleb A. Husseini

Brigham Young University - Provo

Larry Lin Baxter

Brigham Young University - Provo, larry_baxter@byu.edu

Matthew R. Linford

Brigham Young University - Provo

Follow this and additional works at: https://scholarsarchive.byu.edu/facpub

Part of the Chemical Engineering Commons

\section{Original Publication Citation}

Jiang, G., Husseini, G. A., Baxter, L. L., \& Linford, M. R. (2005). Analysis of sawdust by x-ray photoelectron spectroscopy. Surface Science Spectra, 11(1): 127-134.

\section{BYU ScholarsArchive Citation}

Jiang, Guilin; Husseini, Ghaleb A.; Baxter, Larry Lin; and Linford, Matthew R., "Analysis of Sawdust by X-ray Photoelectron Spectroscopy" (2005). Faculty Publications. 1735.

https://scholarsarchive.byu.edu/facpub/1735

This Peer-Reviewed Article is brought to you for free and open access by BYU ScholarsArchive. It has been accepted for inclusion in Faculty Publications by an authorized administrator of BYU ScholarsArchive. For more information, please contact ellen_amatangelo@byu.edu. 


\section{Analysis of Sawdust by X-ray Photoelectron Spectroscopy}

Article in Surface Science Spectra · January 2004

CITATIONS

0

4 authors, including:

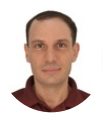

Ghaleb A. Husseini

American University of Sharjah

128 PUBLICATIONS $\quad 1,911$ CITATIONS

SEE PROFILE

Matthew R Linford

Brigham Young University - Provo Main Cam...

305 PUBLICATIONS 5,282 CITATIONS

SEE PROFILE
READS

86
Larry L. Baxter

Brigham Young University - Provo Main Cam...

389 PUBLICATIONS 4,906 CITATIONS

SEE PROFILE 


\section{Analysis of Sawdust by X-ray Photoelectron Spectroscopy}

\author{
Guilin Jiang, Ghaleb A. Husseini, ${ }^{\text {a) }}$ Larry L. Baxter, and Matthew R. Linford \\ Brigham Young University, Provo, Utah 84604
}

(Received 12 August 2004; accepted 16 November 2005; published 30 December 2005)

Determining the chemical structure and composition of biomass fuels using x-ray photoelectron spectroscopy (XPS) can provide fundamental knowledge of their structures that is useful in understanding and predicting their combustion behavior. Sawdust is an example of a forest product residue (byproduct of paper and lumber production) of potential interest for biomass combustion. The XPS spectra of sawdust provide both its elemental composition and indications of its bonding. Traditional fuel analyses of this fuel are also provided. These include: ultimate analysis - the elemental composition of the overall fuel $(\mathrm{C}, \mathrm{H}, \mathrm{N}, \mathrm{S}$, and $\mathrm{O})$; chlorine analysis — reported here as part of the ultimate analysis but formally a separate procedure; proximate analysis - the proximate composition of the fuel (moisture, fixed carbon, volatiles, and ash); heating value - the specific heat of combustion; ash chemistry analysis - an elemental analysis of the ash content, expressed as oxides (which does not imply that they occur as oxides in the fuel). These data are summarized with the XPS spectra. (C) 2005 American Vacuum Society. [DOI: 10.1116/11.20040806]

\author{
Accession \# 00900 \\ Technique: XPS \\ Host Material: sawdust \\ Instrument: Surface Science \\ Instruments SSX-100 \\ Major Elements in Spectrum: C, O \\ Minor Elements in Spectrum: N, Si \\ Printed Spectra: 8 \\ Spectra in Electronic Record: 8 \\ Spectral Category: technical
}

Keywords: biomass; sawdust; XPS; fuel

PACS: 82.80.Pv, 01.30.Kj, 84.60.Rb, 82.60.Cx

\section{SPECIMEN DESCRIPTION}

Host Material: sawdust

Host Material Characteristics: homogeneous; amorphous; unknown electrical characteristics; biological material; powder

Chemical Name: cellulose

Host Composition: see entry for History \& Significance

Form: powder

History \& Significance: Sawdust is an example of a forest product residue (byproduct of paper and lumber production) of potential interest for biomass combustion. Agricultural residues studies here came from 2002 harvests of wood and other agricultural materials in the U.S. and in Europe. The wood came from a commercial saw mill in the western U.S. It is of fairly typical composition of waste materials produced in sawmills. All materials underwent extensive homogenization and particle size classification to produce suitable feed materials for combustion tests conducted in the U.S. Some of the materials were prepared by us and others by collaborators in the U.S. and in Europe.

The XPS spectra of sawdust provide both its elemental composition and indications of its bonding. Traditional fuel analyses of this fuel are also provided. These include: ultimate analysis - the elemental composition of the overall fuel $(\mathrm{C}, \mathrm{H}$, $\mathrm{N}, \mathrm{S}$, and $\mathrm{O}$ ); chlorine analysis — reported here as part of the ultimate analysis but formally a separate procedure; proximate analysis - the proximate composition of the fuel (moisture, fixed carbon, volatiles, and ash); heating value — the specific heat of combustion; ash chemistry analysis — an elemental analysis of the ash content, expressed as oxides (which does not imply they occur as oxides in the fuel). These data are summarized with the XPS spectra.

a) Author to whom correspondence should be addressed; present address: Chemical Engineering Department, P.O. Box 26666, The American University of Sharjah, Sharjah, United Arab Emirates.

\begin{tabular}{lc} 
Table 1: Fuel analysis of bio-fuel sunflower shells \\
(ash free basis except for ash and LHV, which are \\
on an as-received basis). \\
\hline & $\%$ by weight \\
\cline { 2 - 2 } Moisture & 9.84 \\
C & 46.40 \\
H & 5.85 \\
O & 34.33 \\
N & 0.88 \\
S & 0.18 \\
Ash & 2.52 \\
Sum & 100.0 \\
LHV*, MJ/kg & 18.816
\end{tabular}

*Lower heating value

Table 2: Ash composition of sunflower shells (percent of ash basis).

\begin{tabular}{lc}
\hline & Mass $\%$ \\
\cline { 2 - 2 } $\mathrm{SiO}_{2}$ & 1.1 \\
$\mathrm{Al}_{2} \mathrm{O}_{3}$ & 0.5 \\
$\mathrm{Fe}_{2} \mathrm{O}_{3}$ & 0.9 \\
$\mathrm{CaO}$ & 16 \\
$\mathrm{MgO}$ & 13.1 \\
$\mathrm{Na}_{2} \mathrm{O}$ & $<0.2$ \\
$\mathrm{~K}_{2} \mathrm{O}$ & 45.1 \\
$\mathrm{SO}_{3}$ & 11.7 \\
$\mathrm{P}_{2} \mathrm{O}_{5}$ & 10.1 \\
$\mathrm{Cl}$ & 1.2 \\
Other & 0.3 \\
Sum & 100 \\
\hline
\end{tabular}


As Received Condition: powder

Analyzed Region: same as host material

Ex Situ Preparation/Mounting: Sawdust powders were used as received. The powders were pressed onto a piece of nonconductive double-sticky tape mounted on a piece of silicon, which was then mounted on the sample stage with a piece of the same tape.

In Situ Preparation: none

Pre-Analysis Beam Exposure: No damage was observed in the sample even after several hours of exposure to $\mathrm{x}$-ray radiation. After $4 \mathrm{~h}$ of exposure to $\mathrm{x}$ rays, the intensity of the $\mathrm{N} 1 s$ scan did not change.

Charge Control: A flood gun was applied. The flood gun voltage was $4 \mathrm{~V}$, and its current was less than $50 \mathrm{~mA}$. A metal screen was used to mask the sample. The charge control was determined by observing zirconia $\mathrm{Zr} 3 p_{3 / 2}$ peak positions under different flood gun settings. XPS spectra showed a $\mathrm{Zr} 3 d_{5 / 2}$ at $182.3 \mathrm{eV}$. The metal screen used was nickel, $1 \mathrm{~mm}$ distance, 70 lines/in. and $90 \%$ transmission.

Temp. During Analysis: $298 \mathrm{~K}$

Pressure During Analysis: $<2.0 \times 10^{-6} \mathrm{~Pa}$

\section{INSTRUMENT DESCRIPTION}

Manufacturer and Model: Surface Science Instruments SSX-100

Analyzer Type: spherical sector

Detector: resistive anode position detector

Number of Detector Elements: 128

INSTRUMENT PARAMETERS COMMON TO ALL SPECTRA

\section{Spectrometer}

Analyzer Mode: constant pass energy
Throughput $\left(\boldsymbol{T}=\boldsymbol{E}^{N}\right): \quad N=0$

Excitation Source Window: $12 \mu \mathrm{m}$ aluminum foil

Excitation Source: $\mathrm{Al} K_{\alpha}$ monochromatic

Source Energy: $1486.6 \mathrm{eV}$

Source Strength: $200 \mathrm{~W}$

Signal Mode: multichannel direct

\section{Geometry}

Incident Angle: $55^{\circ}$

Source to Analyzer Angle: $70.8^{\circ}$

Emission Angle: $55^{\circ}$

Specimen Azimuthal Angle: $0^{\circ}$

Acceptance Angle from Analyzer Axis: $0^{\circ}$

DATA ANALYSIS METHOD

Peak Shape and Background Method: background Shirley function

Quantitation Method: Sensitivity factors were obtained from ESCA 2000 NT software supplied by Service Physics. The peak areas are the areas above a linear background.

\section{ACKNOWLEDGMENTS}

The authors acknowledge U.S. DOE Biomass Power Program for financial support, and Elsam engineering and Eltra, both Danish companies, which provided complementary analyses and some financial support for this investigation. 
SPECTRAL FEATURES TABLE

\begin{tabular}{|c|c|c|c|c|c|c|c|}
\hline $\begin{array}{l}\text { Spectrum } \\
\text { ID \# }\end{array}$ & $\begin{array}{l}\text { Element/ } \\
\text { Transition }\end{array}$ & $\begin{array}{c}\text { Peak } \\
\text { Energy } \\
(\mathrm{eV})\end{array}$ & $\begin{array}{c}\text { Peak Width } \\
\text { FWHM } \\
(\mathrm{eV})\end{array}$ & $\begin{array}{c}\text { Peak Area } \\
\text { (counts) }\end{array}$ & $\begin{array}{c}\text { Sensitivity } \\
\text { Factor }\end{array}$ & $\begin{array}{l}\text { Concen- } \\
\text { tration } \\
\text { (at. \%) }\end{array}$ & $\begin{array}{c}\text { Peak } \\
\text { Assignment }\end{array}$ \\
\hline 00900-02 & $\mathrm{O} 1 s$ & 528.2 & 3.9 & 88500 & 2.5 & 21.3 & $\cdots$ \\
\hline 00900-03 & $\mathrm{C} 1 s$ & 280.9 & 4.3 & 126000 & 1 & 76 & $\ldots$ \\
\hline 00900-04 & $\mathrm{N} 1 s$ & 396.7 & 5.2 & 16900 & 1.68 & 0.6 & $\cdots$ \\
\hline 00900-05 & Si $2 p$ & 99.3 & 4.8 & 12200 & 0.9 & 2 & $\ldots$ \\
\hline 00900-06 & $\mathrm{O} 1 s$ & 528.3 & 3.5 & 24000 & 2.5 & $\ldots$ & $\ldots$ \\
\hline 00900-07 & C $1 s$ & 281.0 & 4.1 & 32100 & 1 & $\cdots$ & $\cdots$ \\
\hline 00900-08 & $\mathrm{N} 1 s$ & 396.7 & 4.8 & 20300 & 1.68 & $\cdots$ & $\cdots$ \\
\hline
\end{tabular}

\begin{tabular}{|c|c|c|c|c|c|}
\hline \multicolumn{6}{|c|}{ GUIDE TO FIGURES } \\
\hline $\begin{array}{l}\text { Spectrum } \\
\text { (Accession) \# }\end{array}$ & $\begin{array}{l}\text { Spectral } \\
\text { Region }\end{array}$ & $\begin{array}{l}\text { Voltage } \\
\text { Shift }^{\star}\end{array}$ & Multiplier & Baseline & Comment \# \\
\hline $900-1$ & Survey & 0 & 1 & 0 & 1 \\
\hline $900-2$ & $\mathrm{O} 1 s$ & 0 & 1 & 0 & 1 \\
\hline $900-3$ & $\mathrm{C} 1 s$ & 0 & 1 & 0 & 1 \\
\hline $900-4$ & $\mathrm{~N} 1 s$ & 0 & 1 & 0 & 1 \\
\hline $900-5$ & Si $2 p$ & 0 & 1 & 0 & 1 \\
\hline $900-6$ & $\mathrm{O} 1 s$ & 0 & 1 & 0 & 2 \\
\hline $900-7$ & $\mathrm{C} 1 s$ & 0 & 1 & 0 & 2 \\
\hline $900-8$ & $\mathrm{~N} 1 s$ & 0 & 1 & 0 & 2 \\
\hline
\end{tabular}

* Voltage shift of the archived (as-measured) spectrum relative to the printed figure. The figure reflects the recommended energy scale correction due to a calibration correction, sample charging, flood gun, or other phenomenon.

1. $800 \mu \mathrm{m} x$-ray beam diameter, $150 \mathrm{eV}$ pass energy

2. $300 \mu \mathrm{m} x$-ray beam diameter, $50 \mathrm{eV}$ pass energy 


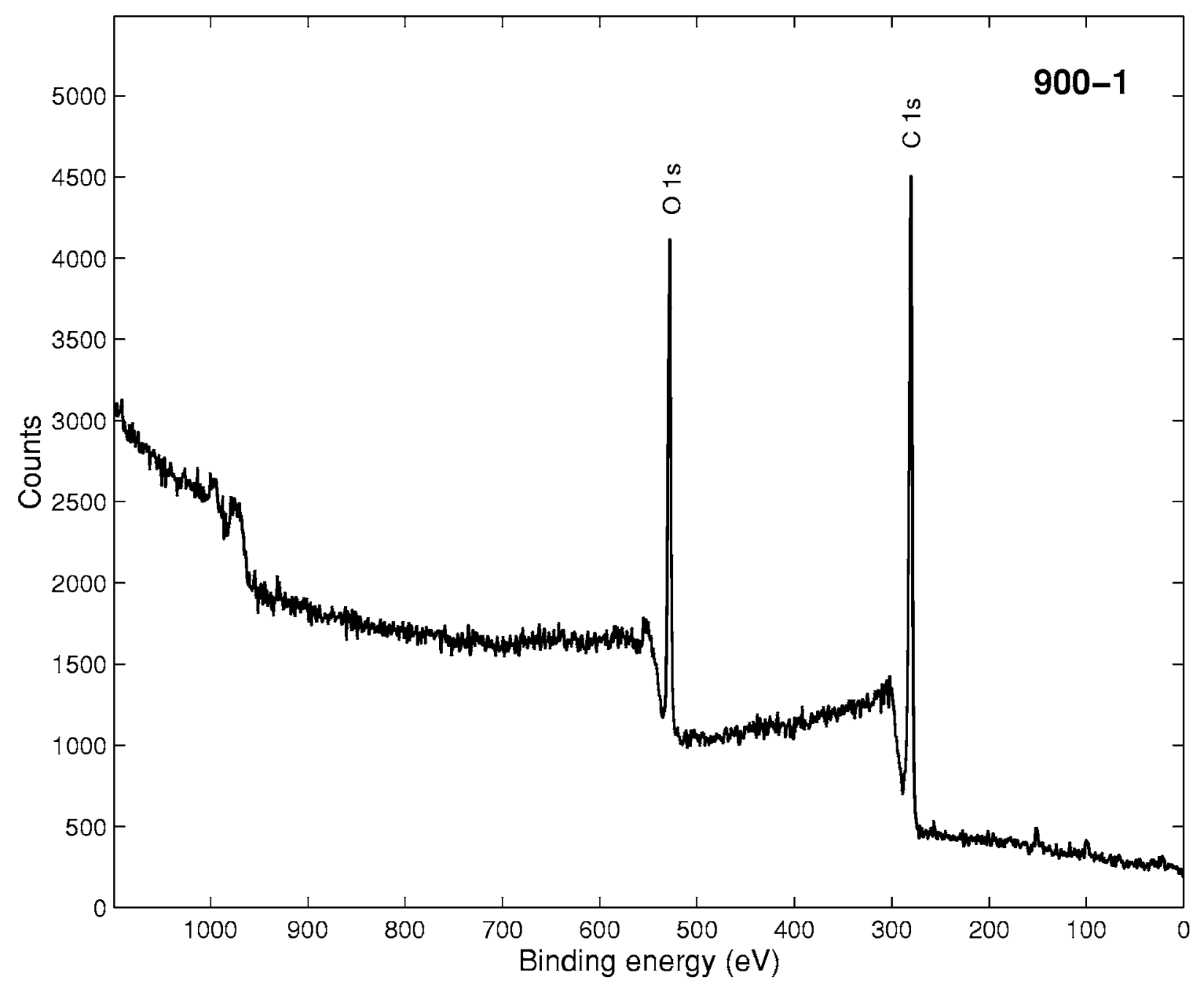

\begin{tabular}{|c|c|}
\hline Accession \# & 00900-01 \\
\hline Host Material & sawdust \\
\hline Technique & XPS \\
\hline Spectral Region & survey \\
\hline Instrument & Surface Science Instruments SSX-100 \\
\hline Excitation Source & Al $K_{\alpha}$ monochromatic \\
\hline Source Energy & $1486.6 \mathrm{eV}$ \\
\hline Source Strength & $200 \mathrm{~W}$ \\
\hline Source Size & $0.8 \mathrm{~mm} \times 0.8 \mathrm{~mm}$ \\
\hline Analyzer Type & spherical sector \\
\hline Incident Angle & $55^{\circ}$ \\
\hline Emission Angle & $55^{\circ}$ \\
\hline Analyzer Pass Energy & $150 \mathrm{eV}$ \\
\hline Analyzer Resolution & $1.5 \mathrm{eV}$ \\
\hline Total Signal Accumulation Time & $2200 \mathrm{~s}$ \\
\hline Total Elapsed Time & $2400 \mathrm{~s}$ \\
\hline Number of Scans & 10 \\
\hline Source Beam Size at Specimen Surface & $0.8 \mathrm{~mm} \times 1.392 \mathrm{~mm}$ \\
\hline Effective Detector Width & $19 \mathrm{eV}$ \\
\hline Analyzer Width & $1500 \mu \mathrm{m} \times 12000 \mu \mathrm{m}$ at $84 \mathrm{eV}$ \\
\hline
\end{tabular}




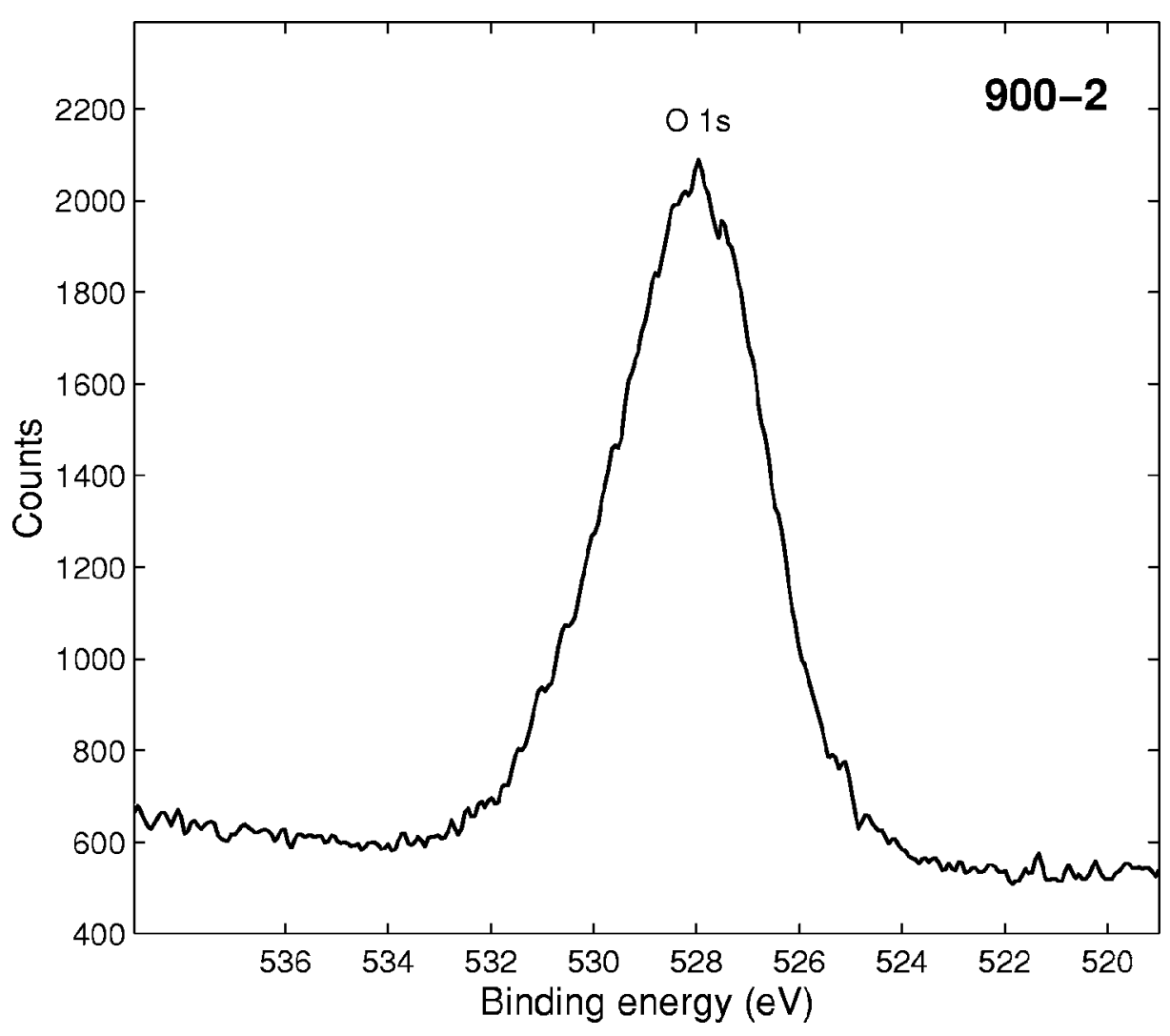

- Accession \#: 00900-02

- Host Material: sawdust

- Technique: XPS

- Spectral Region: $01 \mathrm{~s}$

Instrument: Surface Science Instruments SSX-100

Excitation Source: Al $K_{\alpha}$ monochromatic

Source Energy: $1486.6 \mathrm{eV}$ Source Strength: $200 \mathrm{~W}$

Source Size: $0.8 \mathrm{~mm} \times 0.8 \mathrm{~mm}$ Incident Angle: $55^{\circ}$

Analyzer Type: spherical sector Analyzer Pass Energy: $150 \mathrm{eV}$ Analyzer Resolution: $1.5 \mathrm{eV}$

Emission Angle: $55^{\circ}$

Total Signal Accumulation Time: $306.5 \mathrm{~s}$

Total Elapsed Time: $475.5 \mathrm{~s}$

Number of Scans: 5

Source Beam Size at Specimen Surface: $0.8 \mathrm{~mm} \times 1.392 \mathrm{~mm}$ Effective Detector Width: $19 \mathrm{eV}$ Analyzer Width: $1500 \mu \mathrm{m} \times$ $12000 \mu \mathrm{m}$ at $84 \mathrm{eV}$

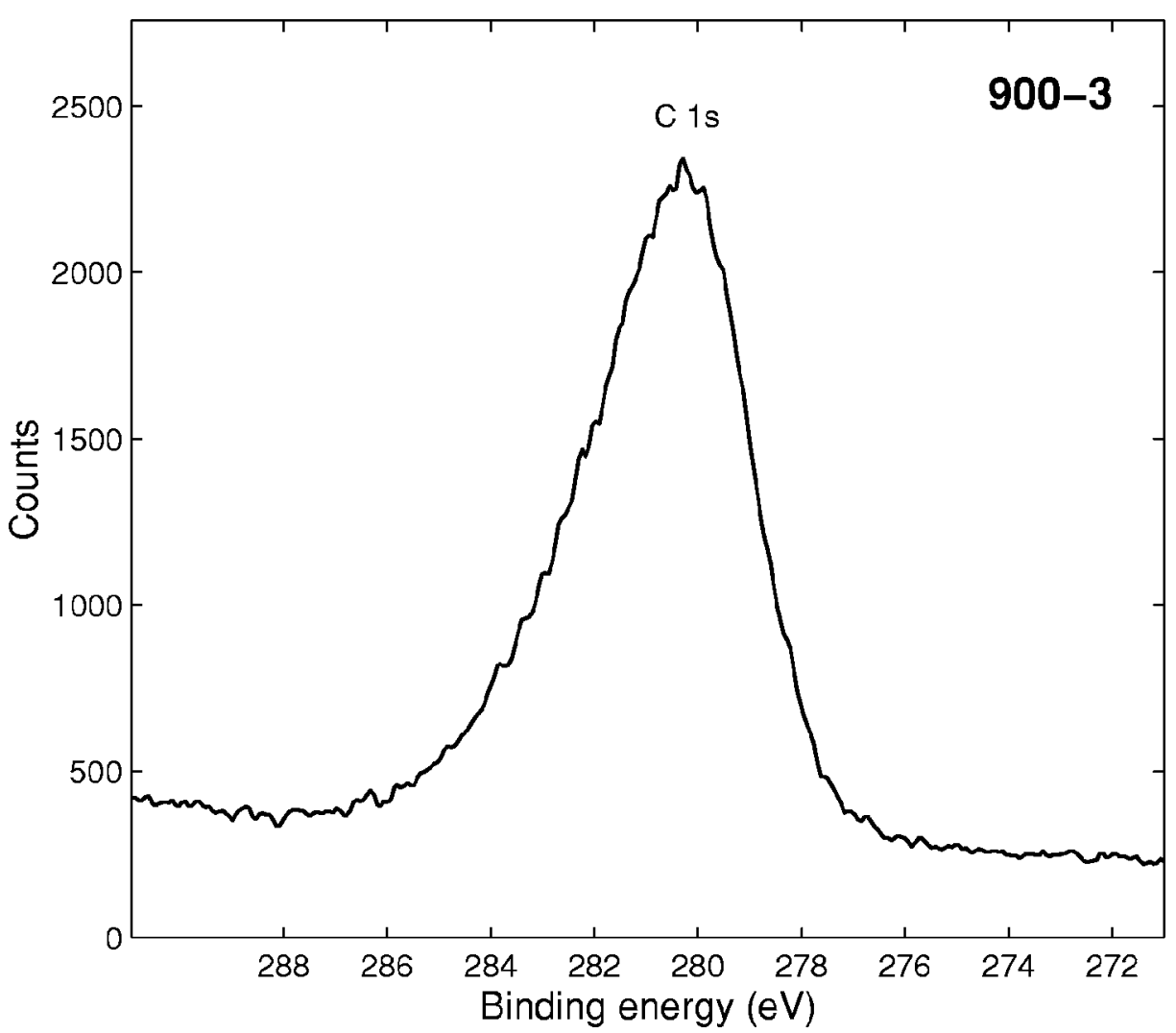

- Accession \#: 00900-03

- Host Material: sawdust

- Technique: XPS

- Spectral Region: C $1 \mathrm{~s}$

Instrument: Surface Science Instruments SSX-100

Excitation Source: Al $K_{\alpha}$ monochromatic

Source Energy: $1486.6 \mathrm{eV}$

Source Strength: $200 \mathrm{~W}$

Source Size: $0.8 \mathrm{~mm} \times 0.8 \mathrm{~mm}$ Incident Angle: $55^{\circ}$

Analyzer Type: spherical sector Analyzer Pass Energy: $150 \mathrm{eV}$ Analyzer Resolution: $1.5 \mathrm{eV}$

Emission Angle: $55^{\circ}$

Total Signal Accumulation Time: $306.5 \mathrm{~s}$

Total Elapsed Time: $475.5 \mathrm{~s}$

Number of Scans: 5

Source Beam Size at Specimen Surface: $0.8 \mathrm{~mm} \times 1.392 \mathrm{~mm}$ Effective Detector Width: $19 \mathrm{eV}$ Analyzer Width: $1500 \mu \mathrm{m} \times$ $12000 \mu \mathrm{m}$ at $84 \mathrm{eV}$ 


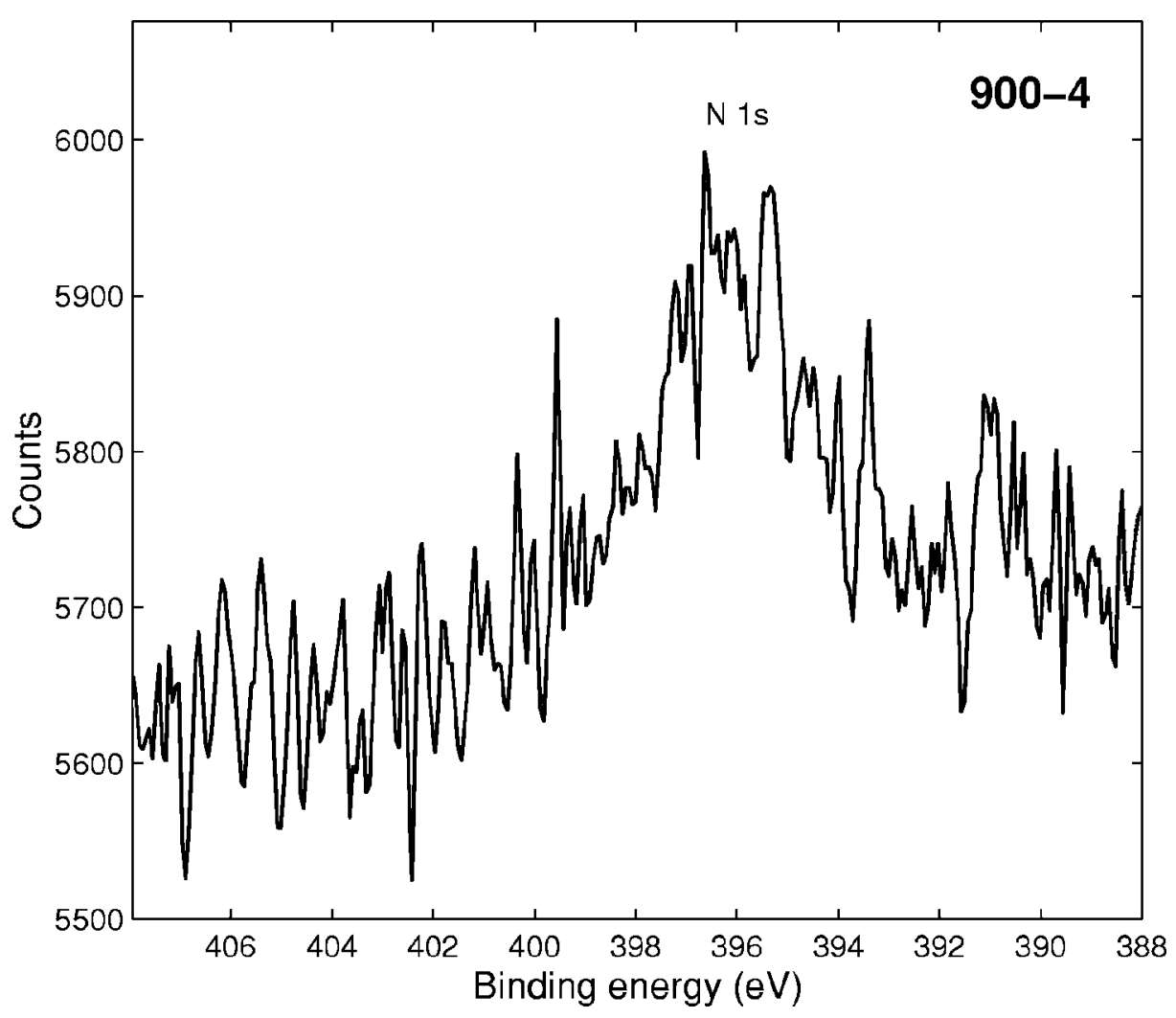

- Accession \#: 00900-04

- Host Material: sawdust

- Technique: XPS

- Spectral Region: N1s

Instrument: Surface Science Instruments SSX-100

Excitation Source: $\mathrm{Al} K_{\alpha}$ monochromatic

Source Energy: $1486.6 \mathrm{eV}$

Source Strength: $200 \mathrm{~W}$

Source Size: $0.8 \mathrm{~mm} \times 0.8 \mathrm{~mm}$ Incident Angle: $55^{\circ}$

Analyzer Type: spherical sector Analyzer Pass Energy: $150 \mathrm{eV}$

Analyzer Resolution: $1.5 \mathrm{eV}$

Emission Angle: $55^{\circ}$

Total Signal Accumulation Time: $3065 \mathrm{~s}$

Total Elapsed Time: $3234 \mathrm{~s}$

Number of Scans: 50

Source Beam Size at Specimen Surface: $0.8 \mathrm{~mm} \times 1.392 \mathrm{~mm}$ Effective Detector Width: $19 \mathrm{eV}$ Analyzer Width: $1500 \mu \mathrm{m} \times$ $12000 \mu \mathrm{m}$ at $84 \mathrm{eV}$

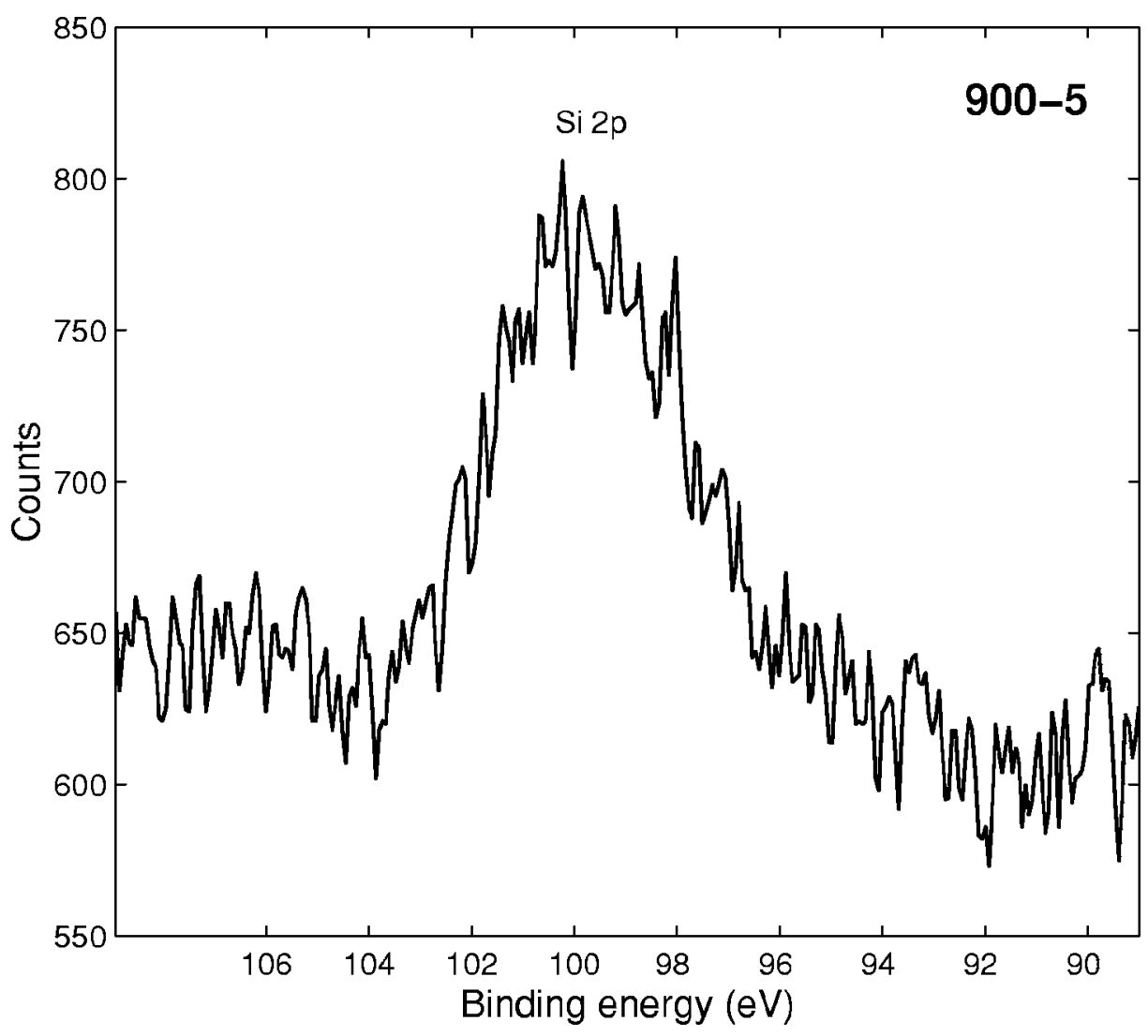

- Accession \#: 00900-05

- Host Material: sawdust

- Technique: XPS

- Spectral Region: Si $2 p$

Instrument: Surface Science Instruments SSX-100

Excitation Source: Al $K_{\alpha}$ monochromatic

Source Energy: $1486.6 \mathrm{eV}$

Source Strength: $200 \mathrm{~W}$

Source Size: $0.8 \mathrm{~mm} \times 0.8 \mathrm{~mm}$ Incident Angle: $55^{\circ}$

Analyzer Type: spherical sector Analyzer Pass Energy: $150 \mathrm{eV}$ Analyzer Resolution: $1.5 \mathrm{eV}$

Emission Angle: $55^{\circ}$

Total Signal Accumulation Time: $1226 \mathrm{~s}$

Total Elapsed Time: $1395 \mathrm{~s}$

Number of Scans: 20

Source Beam Size at Specimen Surface: $0.8 \mathrm{~mm} \times 1.392 \mathrm{~mm}$ Effective Detector Width: $19 \mathrm{eV}$ Analyzer Width: $1500 \mu \mathrm{m} \times$ $12000 \mu \mathrm{m}$ at $84 \mathrm{eV}$ 


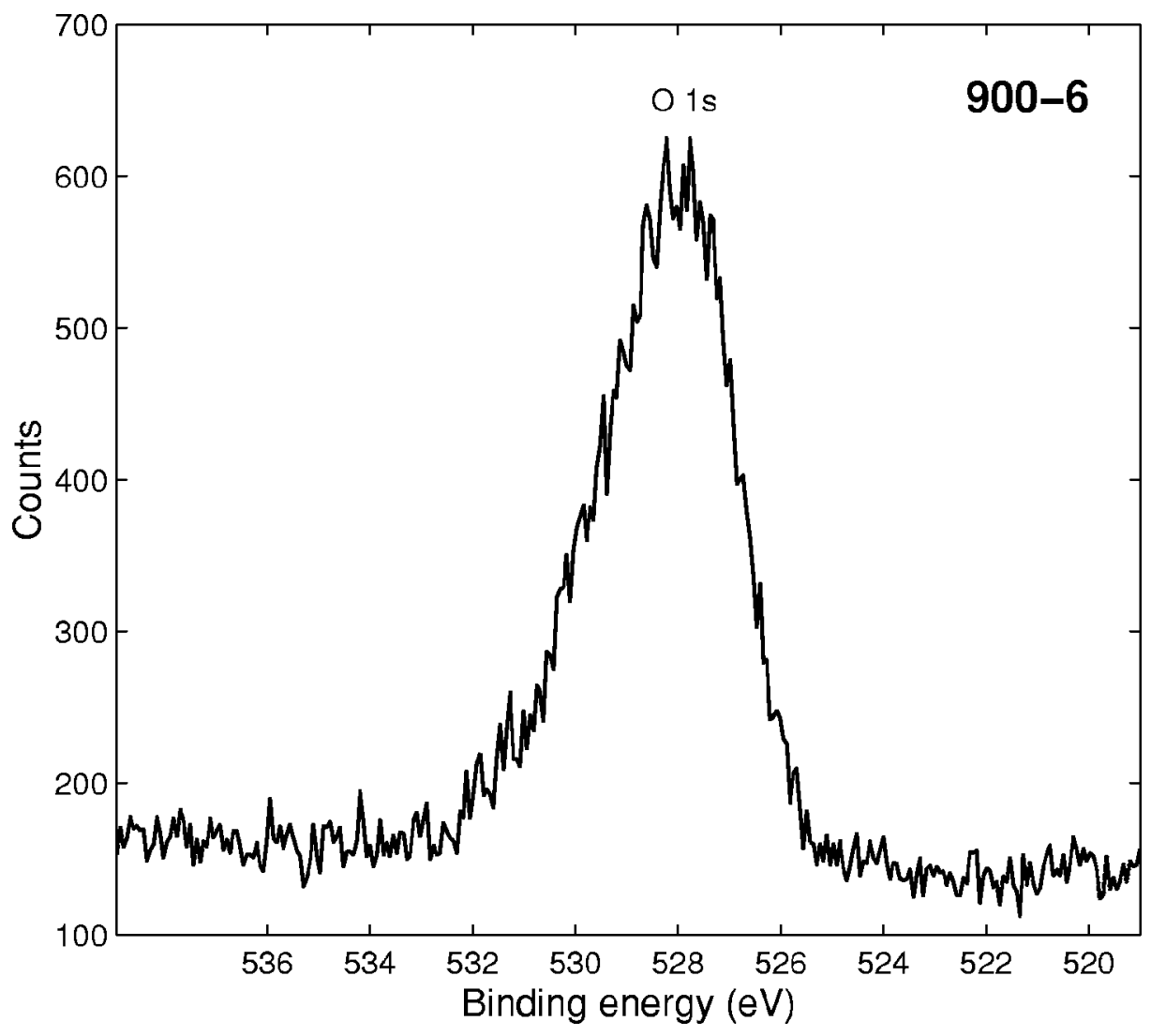

- Accession \#: 00900-06

- Host Material: sawdust

- Technique: XPS

- Spectral Region: $01 \mathrm{~s}$

Instrument: Surface Science Instruments SSX-100

Excitation Source: Al $K_{\alpha}$ monochromatic

Source Energy: $1486.6 \mathrm{eV}$ Source Strength: $200 \mathrm{~W}$

Source Size: $0.3 \mathrm{~mm} \times 0.3 \mathrm{~mm}$ Incident Angle: $55^{\circ}$

Analyzer Type: spherical sector Analyzer Pass Energy: $50 \mathrm{eV}$ Analyzer Resolution: $0.5 \mathrm{eV}$

Emission Angle: $55^{\circ}$

Total Signal Accumulation Time: $613 \mathrm{~s}$

Total Elapsed Time: $782 \mathrm{~s}$

Number of Scans: 10

Source Beam Size at Specimen Surface: $0.3 \mathrm{~mm} \times 0.523 \mathrm{~mm}$ Effective Detector Width: $6.6 \mathrm{eV}$ Analyzer Width: $750 \mu \mathrm{m} \times$ $6000 \mu \mathrm{m}$ at $84 \mathrm{eV}$

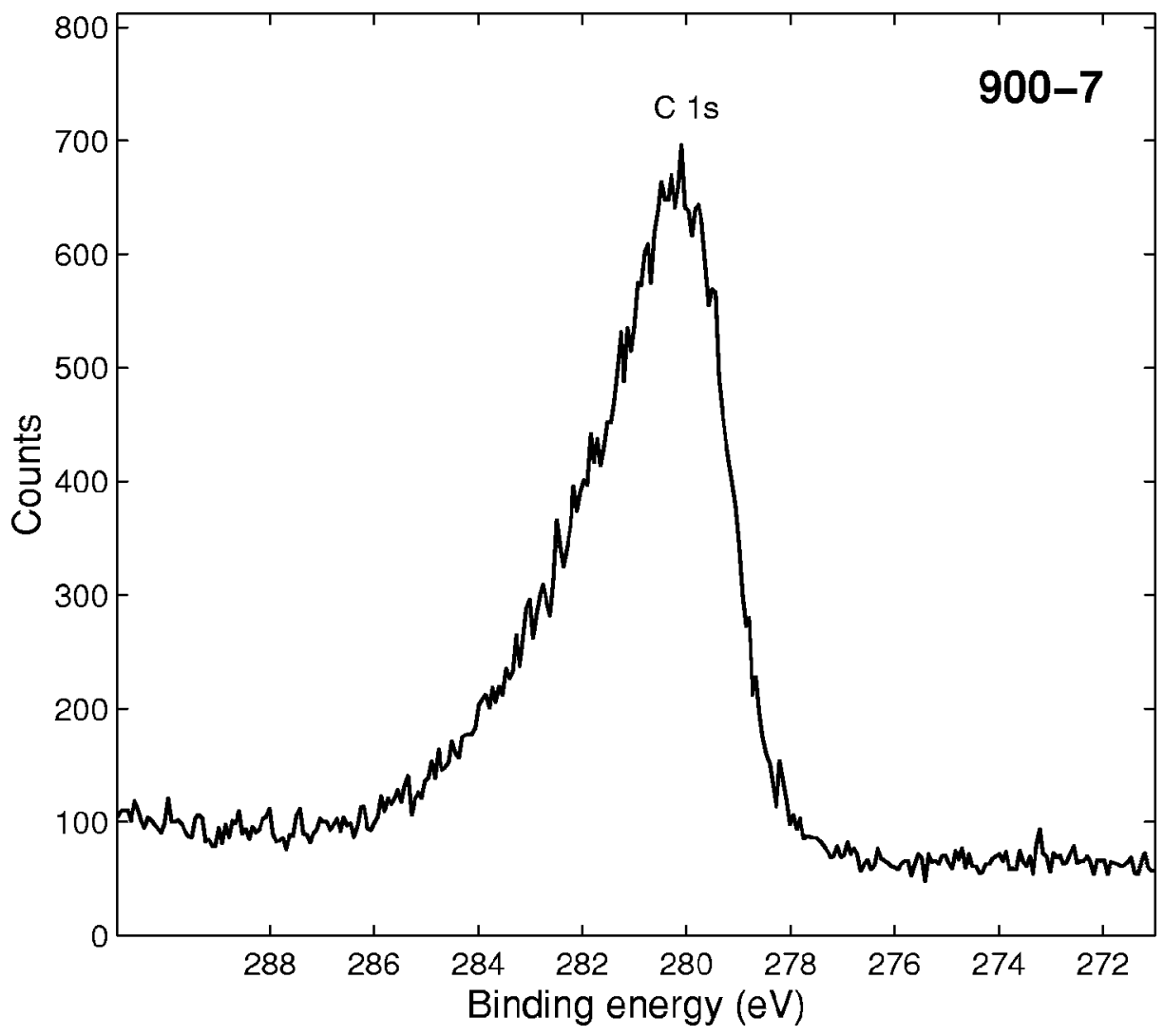

- Accession \#: 00900-07

- Host Material: sawdust

- Technique: XPS

- Spectral Region: C $1 \mathrm{~s}$

Instrument: Surface Science Instruments SSX-100

Excitation Source: Al $K_{\alpha}$ monochromatic

Source Energy: $1486.6 \mathrm{eV}$

Source Strength: $200 \mathrm{~W}$

Source Size: $0.3 \mathrm{~mm} \times 0.3 \mathrm{~mm}$ Incident Angle: $55^{\circ}$

Analyzer Type: spherical sector Analyzer Pass Energy: $50 \mathrm{eV}$ Analyzer Resolution: $0.5 \mathrm{eV}$

Emission Angle: $55^{\circ}$

Total Signal Accumulation Time: $613 \mathrm{~s}$

Total Elapsed Time: $782 \mathrm{~s}$

Number of Scans: 10

Source Beam Size at Specimen Surface: $0.3 \mathrm{~mm} \times 0.523 \mathrm{~mm}$ Effective Detector Width: $6.6 \mathrm{eV}$ Analyzer Width: $750 \mu \mathrm{m} \times$ $6000 \mu \mathrm{m}$ at $84 \mathrm{eV}$ 


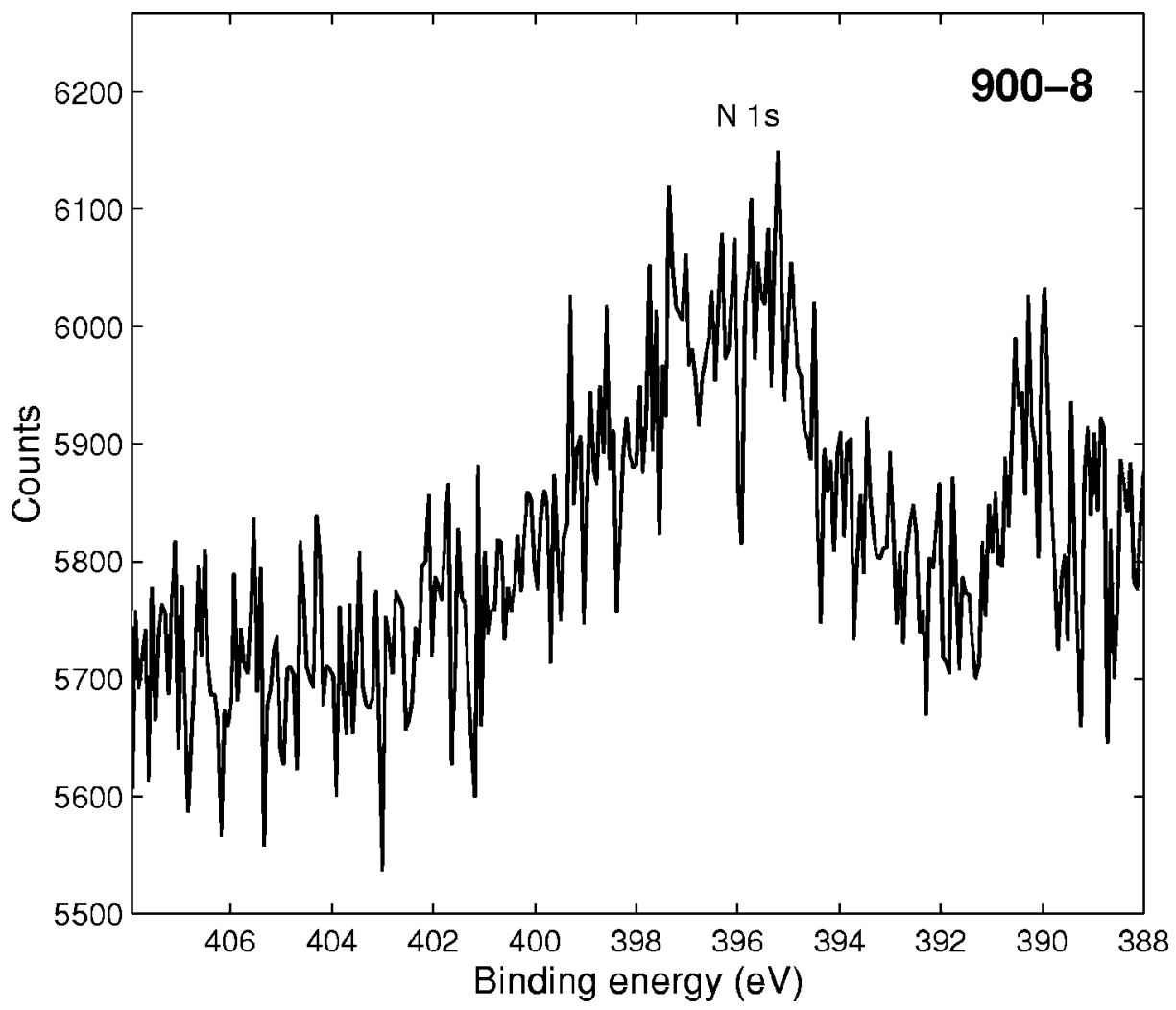

- Accession \#: 00900-08

- Host Material: sawdust

- Technique: XPS

- Spectral Region: N1s

Instrument: Surface Science Instruments SSX-100

Excitation Source: $\mathrm{Al} K_{\alpha}$ monochromatic

Source Energy: $1486.6 \mathrm{eV}$

Source Strength: $200 \mathrm{~W}$

Source Size: $0.3 \mathrm{~mm} \times 0.3 \mathrm{~mm}$

Incident Angle: $55^{\circ}$

Analyzer Type: spherical sector Analyzer Pass Energy: $50 \mathrm{eV}$

Analyzer Resolution: $0.5 \mathrm{eV}$

Emission Angle: $55^{\circ}$

Total Signal Accumulation Time: $24520 \mathrm{~s}$

Total Elapsed Time: $24689 \mathrm{~s}$

Number of Scans: 400

Source Beam Size at Specimen Surface: $0.3 \mathrm{~mm} \times 0.523 \mathrm{~mm}$ Effective Detector Width: $6.6 \mathrm{eV}$ Analyzer Width: $750 \mu \mathrm{m} \times$ $6000 \mu \mathrm{m}$ at $84 \mathrm{eV}$ 